\title{
New ways in which GLP-1 can regulate glucose homeostasis
}

\author{
David A. D'Alessio, Darleen A. Sandoval, and Randy J. Seeley
}

Departments of Medicine and Psychiatry, Genome Research Institute, University of Cincinnati, Cincinnati, Ohio, USA.

\begin{abstract}
Glucagon-like peptide-1 (GLP-1) has a diverse set of peripheral actions which all serve to promote enhanced glucose tolerance, and for this reason it has become the basis for new treatments for type 2 diabetes. In this issue of the JCI, Knauf et al. provide clear evidence that GLP-1 signaling in the CNS is also linked to the control of peripheral glucose homeostasis by inhibiting non-insulin-mediated glucose uptake by muscle and increasing insulin secretion from the pancreas (see the related article beginning on page 3554). The authors' work points to an important need to integrate diverse GLP-1 signaling actions and peripheral GLP-1 function in order to better understand both normal and abnormal glucose homeostasis.
\end{abstract}

Glucagon-like peptide-1 (GLP-1) is a brain-gut peptide that has enjoyed considerable attention recently because of its important role in the regulation of glucose homeostasis and its application to the treatment of diabetes. GLP-1 was first defined as an incretin, a gastrointestinal hormone released during meal absorption that stimulates prandial insulin release (1). However, subsequent studies demonstrated that intravenous administration of GLP-1 has effects on glucagon secretion, gastric function, hepatic glucose metabolism, and satiety (2). This expanded set of functions was also attributed to endocrine effects of intestinally derived GLP-1. Based on current evidence, the actions of circulating GLP- 1 are best understood as a set of coordinated processes that promotes glucose tolerance (Figure 1). Indeed, the most consistent finding across disparate studies in which GLP-1 signaling was blocked or reduced in vivo has been the development of abnormally high blood glucose levels following enteral or parenteral glucose administration (3-6).

\section{The CNS GLP-1 system}

Besides the gut, the other major site of GLP-1 production is the brain. GLP-1 is produced in a discrete set of hindbrain neu-

Nonstandard abbreviations used: GLP-1, glucagonlike peptide-1.

Conflict of interest: The authors have declared that no conflict of interest exists.

Citation for this article: J. Clin. Invest. 115:3406-3408 (2005). doi:10.1172/JCI27207. rons that project to specific populations of GLP-1 receptor-containing cells in the brain stem, hypothalamus, and midbrain. These circuits have been implicated in food intake (7), response to illness (8), and mediation of stress $(9,10)$. While a rapidly growing body of research has established the importance of GLP-1 signaling in the brain, most of this work has suggested that CNS and peripheral actions of GLP-1 are distinct and independent.

In this issue of the JCI, Knauf and colleagues present a series of novel findings that challenge the current understanding of GLP-1 physiology (11). In a series of technically demanding experiments, they demonstrate that administration of a GLP-1 receptor antagonist into the cerebroventricular system of mice increased total body glucose disposal, with increased formation of glycogen in skeletal muscle and reduced hepatic glycogen levels. These effects were also found in mice with a knockout of the GLP-1 receptor and were reduced by selective muscle denervation, suggesting that GLP-1 activity in the brain initiates peripheral neural signals that regulate systemic glucose metabolism. The CNS GLP-1 receptor antagonist-mediated increase in glucose disposal was proportional to ambient glycemia and independent of muscle insulin action, findings that are consistent with previous demonstrations that non-insulin-mediated glucose uptake is driven by mass action (12). Interestingly, when CNS GLP-1 receptors were blocked, there was a significant attenuation of mealinduced insulin secretion (11). Based on their findings, the authors propose that during the hyperglycemia that follows a meal, CNS GLP-1 inhibits muscle glucose utilization and increases insulin secretion to favor hepatic glycogen storage, improving the body's preparation for the next fasting state. This study is important because of the novel demonstration that central GLP-1 signaling appears to be connected to the control of blood glucose level. However, some uncertainty still remains regarding our ability to construct a unified model of GLP-1 action on glucose homeostasis.

\section{The fate of glucose}

It is generally thought that following glucose ingestion, approximately one-third of the absorbed glucose is distributed to the liver, one-third is distributed to the skeletal muscle and adipose tissue, and the remainder is distributed to non-insulin-dependent tissues including the CNS (13). This distribution is determined by peripheral hormone secretion, CNS signals, and the actual circulating levels of glucose (Figure 1). There is some evidence that enterally absorbed glucose disproportionately increases hepatic glucose uptake compared with intravenous glucose given peripherally (13). It has also been shown that infusion of glucose directly into the hepatic portal vein mimics the effect of an oral glucose load in conscious dogs (14). The effect of a portal vein-arterial glucose gradient to promote glucose storage in the liver has been termed the portal signal. The portal signal has also been shown to stimulate pancreatic insulin secretion (15) and suppress skeletal muscle glucose uptake (16), and the responses stimulated by hepatoportal hyperglycemia have recently been linked to sympathetic nervous system activity (17). It is interesting to note that the current data indicate a similar role for GLP-1 signaling, making it possible that the CNS GLP-1 system acts in parallel with or mediates the portal signal.

Although not studied in great detail, previous reports suggest that intracerebroventricular administration of GLP-1 reduces plasma glucose levels (18). In order to control glycemic stimuli among experimental 


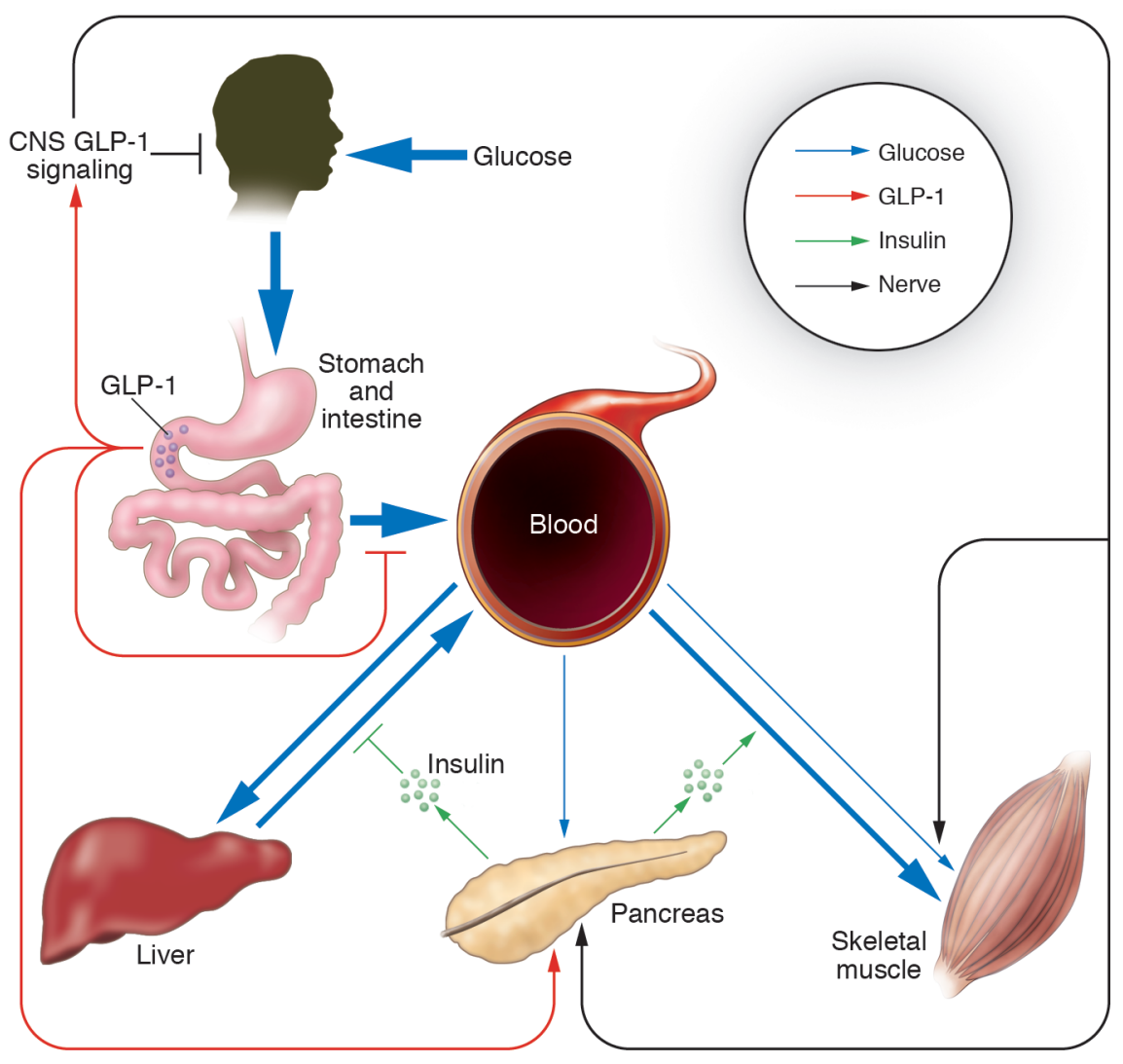

treatments, Knauf et al. (11) clamped glucose, obscuring the net effect of their interventions on actual levels of glucose in the plasma. The model proposed by the authors predicts an increase in CNS GLP-1 activity after meals and a consequent reduction in non-insulin-mediated glucose uptake in muscle, making more glucose available for hepatic glycogen synthesis. However, it is not clear whether this effect on the partitioning of glucose among different tissues would contribute to better glucose tolerance after meals. It does appear that more CNS GLP-1 signaling will increase insulin-mediated glucose disposal by enhancing insulin secretion. But the data presented, showing a dramatic increase in systemic glucose disposal when central GLP-1 receptors were blocked, could be interpreted as showing that increased GLP-1 signaling in the CNS actually impairs glucose tolerance. Thus, it will be crucial for future studies to address the net role of the CNS GLP-1 system in the regulation of blood glucose levels during oral glucose tolerance tests or meals.

\section{Remaining questions}

The supposition of Knauf et al. (11) that brain GLP-1 activity is increased after meals is reasonable based on the effects of the GLP-1 mimetic, exendin 4, during intravenous and enteral hyperglycemia. Central administration of this GLP-1 receptor agonist dramatically increased insulin secretion, insulin-mediated glucose uptake, and hepatic glycogen synthesis when given during an intravenous glucose clamp. However, when administered while blood glucose was raised by an intragastric glucose infusion, the effects of exendin 4 on glucose disposal, insulin secretion, and liver glycogen levels did not differ from controls. In contrast, GLP-1 receptor antagonism increased glucose disposal during both intravenously and enterally induced hyperglycemia. These results suggest that CNS GLP-1 activity is present during the fasting state but increases substantially during gastrointestinal glucose absorption, since it cannot be further enhanced by exogenous exendin 4. Although the observations of Knauf et al. support this implication (11), it is the CNS is not a uniform process. Rather, GLP-1 receptors are distributed in specific brain regions, where it appears that they control distinct functions $(9,19)$. Thus, a major question raised by the present observations (11) is the location of the key important to note that GLP-1 signaling in

\section{Figure 1}

Levels of glucose in the plasma are determined by the rate at which glucose emerges from the gastrointestinal tract as well as the rate of glucose production by the liver. Glucose serves as a stimulus for insulin secretion from the pancreas, which influences the rate of glucose uptake by both liver and muscle. GLP-1 secreted by the intestine inhibits glucose appearance by inhibiting both nutrient intake and gastric emptying rates while stimulating insulin secretion to facilitate muscle and liver glucose uptake. In this issue of the $\mathrm{JCl}$, Knauf et al. (11) provide compelling evidence that GLP-1 signaling in the CNS also serves to increase non-insulin-dependent glucose uptake by muscle and stimulates insulin secretion via neural connections to these tissues. populations of GLP-1 receptors regulating glucose uptake and insulin secretion.

An important related question is how the central GLP-1 system is activated during meals. Previous work has shown that GLP-1expressing neurons in the nucleus of the solitary tract of rats are activated by noxious stimuli, such as lithium chloride and lipopolysaccharide as well as gastric distention, but not normal feeding (20). It is possible that GLP-1 cells in the hindbrain are activated by afferent nerves from the viscera or hepatic portal bed. Alternatively, it may be that case that other factors released during meal absorption initiate central GLP-1 action; cholecystokinin has been shown to have such an effect (20). Finally, it is possible that peripherally released GLP-1 can act in the brain through circumventricular organs (10) or potentially after transport across the blood-brain barrier.

The role of central GLP-1 receptor signaling in glucose tolerance, the neurons important for regulating blood glucose disposition, and the mechanism by which they are activated are critical questions raised by the observations of Knauf et al. (11). The answers to these questions will ultimately help researchers to determine how CNS GLP-1 secretion and function interacts with 
peripheral GLP-1 action. Models and experimental paradigms that permit the dissociation of specific functions of the peripheral and CNS GLP-1 systems will be of great help in this regard. A major impetus for the study of GLP-1 has been the demonstration that peripheral GLP-1 administration is very effective in reducing elevated blood glucose levels in humans with diabetes (21). In fact, the GLP-1 receptor signaling system has become an important target in drug development, with several novel compounds that target this receptor emerging onto the market (22). With our growing appreciation of the important role the CNS plays in both normal and impaired glucose tolerance, the current study makes it imperative that we pursue a detailed understanding of how the CNS GLP-1 system contributes to glucose homeostasis.

Address correspondence to: Randy J. Seeley, Department of Psychiatry, Genome Research Institute, 2170 East Galbraith Road, University of Cincinnati, Cincinnati, Ohio 45267, USA. Phone: (513) 558-6664; Fax: (513) 5588990; E-mail: randy.seeley@uc.edu.

1. Kreymann, B., Ghatei, M.A., Williams, G., and Bloom, S.R. 1987. Glucagon-like peptide-1 7-36: a physiological incretin in man. Lancet. 2:1300-1303. 2. Nauck, M.A., et al. 1993. Normalization of fasting hyperglycaemia by exogenous glucagon-like peptide 1 (7-36 amide) in type 2 (non-insulin-dependent) diabetic patients. Diabetologia. 36:741-744.

3. Wang, Z., et al. 1995. Glucagon-like peptide-1 is a physiological incretin in rat. J. Clin. Invest. 95:417-421.

4. Kolligs, F., Fehmann, H.C., Goke, R., and Goke, B. 1995. Reduction of the incretin effect in rats by the glucagon-like peptide 1 receptor antagonist exendin (9-39) amide. Diabetes. 44:16-19.

5. D'Alessio, D.A., et al. 1996. Elimination of the action of glucagon-like peptide 1 causes an impairment of glucose tolerance after nutrient ingestion by healthy baboons. J. Clin. Invest. 97:133-138.

6. Edwards, C.M., et al. 1999. Glucagon-like peptide 1 has a physiological role in the control of postprandial glucose in humans: studies with the antagonist exendin 9-39. Diabetes. 48:86-93.

7. Turton, M.D., et al. 1996. A role for glucagon-like peptide- 1 in the central regulation of feeding. Nature. 379:69-72.

8. Seeley, R.J., et al. 2000. The role of CNS GLP-1-(7-36) amide receptors in mediating the visceral illness effects of lithium chloride. J. Neurosci. 20:1616-1621.

9. Kinzig, K.P., et al. 2003. CNS glucagon-like peptide-1 receptors mediate endocrine and anxiety responses to interoceptive and psychogenic stressors. J. Neurosci. 23:6163-6170.

10. Yamamoto, H., et al. 2002. Glucagon-like peptide-1 receptor stimulation increases blood pressure and heart rate and activates autonomic regulatory neurons. J. Clin. Invest. 110:43-52. doi:10.1172/ JCI200215595.

11. Knauf, C., et al. 2005. Brain glucagon-like peptide- 1 increases insulin secretion and muscle insulin resistance to favor hepatic glycogen storage. J. Clin. Invest. 115:3554-3563. doi:10.1172/ JCI25764.

12. Best, J.D., Taborsky, G.J., Jr., Halter, J.B., and Porte, D., Jr. 1981. Glucose disposal is not proportional to plasma glucose level in man. Diabetes. 30:847-850.
13. Cherrington, A.D. 1999. Banting Lecture 1997. Control of glucose uptake and release by the liver in vivo. Diabetes. 48:1198-1214.

14. Bergman, R.N., Beir, J.R., and Hourigan, P.M. 1982. Intraportal glucose infusion matched to oral glucose absorption. Lack of evidence for "gut factor" involvement in hepatic glucose storage. Diabetes. 31:27-35.

15. Dunning, B.E., et al. 2002. Portal glucose infusion exerts an incretin effect associated with changes in pancreatic neural activity in conscious dogs. Metabolism. 51:1324-1330.

16. Galassetti, P., Shiota, M., Zinker, B.A., Wasserman, D.H., and Cherrington, A.D. 1998. A negative arterial-portal venous glucose gradient decreases skeletal muscle glucose uptake. Am. J. Physiol. Endocrinol. Metab. 275:E101-E111.

17. DiCostanzo, C.A., et al. 2005. The role of the hepatic sympathetic nerves in the regulation of net hepatic glucose uptake and the mediation of the portal glucose signal. Am. J. Physiol. Endocrinol. Metab. doi:10.1152/ajpendo.00184.2005.

18. Larsen, P.J., Tang-Christensen, M., and Jessop, D.S. 1997. Central administration of glucagon-like peptide- 1 activates hypothalamic neuroendocrine neurons in the rat. Endocrinology. 138:4445-4455.

19. Kinzig, K.P., D’Alessio, D.A., and Seeley, R.J. 2002. The diverse roles of CNS GLP-1 in the control of food intake and the mediation of visceral illness. J. Neurosci. 22:10470-10476.

20. Rinaman, L. 1999. Interoceptive stress activates glucagon-like peptide- 1 neurons that project to the hypothalamus. Am. J. Physiol. 277:R582-R590.

21. Zander, M., Madsbad, S., Madsen, J.L., and Holst, J.J. 2002. Effect of 6-week course of glucagon-like peptide 1 on glycaemic control, insulin sensitivity, and beta-cell function in type 2 diabetes: a parallelgroup study. Lancet. 359:824-830.

22. Ahren, B., and Schmitz, O. 2004. GLP-1 receptor agonists and DPP-4 inhibitors in the treatment of type 2 diabetes. Horm. Metab. Res. 36:867-876. 